\title{
Application of Polysomnography in Nursing Care of Patients with OSAHS
}

\author{
Fang $\mathrm{Xu}^{1}$, Zejun Zhou ${ }^{2, *}$ \\ ${ }^{1}$ Medical Examination Department, the First Affiliated Hospital of Jinan University, Guangzhou, P.R. China \\ ${ }^{2}$ Otolaryngology Department, the First Affiliated Hospital of Jinan University, Guangzhou, P.R. China
}

Email address:

48458659@qq.com (Zejun Zhou)

${ }^{*}$ Corresponding author

\section{To cite this article:}

Fang Xu, Zejun Zhou. Application of Polysomnography in Nursing Care of Patients with OSAHS. American Journal of Biomedical and Life Sciences. Vol. 6, No. 4, 2018, pp. 85-89. doi: 10.11648/j.ajbls.20180604.14

Received: August 13, 2018; Accepted: October 16, 2018; Published: October 18, 2018

\begin{abstract}
To observe the effect of comprehensive nursing intervention on the success rate of polysomnography (PSG) in monitoring (OSAHS) patients with sleep apnea hypopnea syndrome. 240 suspected OSAHS patients were randomly divided into two groups, 120 cases in each group. After admission, all patients were monitored by polysomnography. In the monitoring process, the control group according to the routine nursing methods, the observation group was given peri-monitoring nursing intervention at the same time, the diagnosis rate of OSAHS, monitoring success rate, comfort, emotional changes and satisfaction with nursing were observed. The positive rate of OSAHS in the observation group was 96.7\% (116/120), which was significantly higher than that in the control group $(95.0 \%(114 / 120)$ and $57.0 \%(65 / 114) \mathrm{P}<0.05)$. In the control group, 4 cases $(3.3 \%)$ had shorter sleep time than 2 cases in the observation group $(\mathrm{P}<0.05)$, and the GCQ scores of the observation group were $(46.2 \pm 5.2)$ and $(53.6 \pm 4.1)$ at the beginning of monitoring and at the end of monitoring, respectively, which were higher than those in the control group $(\mathrm{P}<0.05)$. The nursing satisfaction in the observation group $(95.0 \%)$ was significantly higher than that in the control group $(85.8 \%)(\mathrm{P}<0.05)$. The comprehensive nursing intervention in the peri-monitoring period can effectively improve the success rate of PSG in monitoring OSAHS and improve the comfort of the patients during the monitoring process, thus improving the degree of satisfaction of the patients with the nursing work.
\end{abstract}

Keywords: Nursing Intervention, Polysomnography, Sleep Apnea Hypopnea Syndrome, Success Rate

\section{Introduction}

Obstructive sleep apnea hypopnea syndrome (OSAHS) is a kind of respiratory regulation disorder, which is mainly characterized by dyspnea, which usually occurs in nocturnal sleep, leading to anoxia, disorder of metabolic function and impairment of multiple organ functions [1]. Early diagnosis and treatment is the key to improve prognosis [2]. The standard of clinical diagnosis of OSAHS is still polysomnography (PSG) monitoring, multi-dimensional auxiliary monitoring, combined with multiple accurate diagnostic accuracy of data, and the degree of OSAHS can be directly judged III [3]. However, the monitoring time of PSG is long, and it is affected by many aspects, which results in the failure of monitoring sometimes and reduces the diagnostic accuracy [4]. Nursing work plays an important role in the monitoring process. The omnidirectional nursing intervention in peri-monitoring period can improve the patient's cognition, compliance and adaptability, avoid the abnormal electrode conduction, and improve the monitoring success rate [5].

\section{Clinical Data}

\subsection{The General Data}

The general data were numbered with the number of hospitalized medical records. According to the random number table, 240 cases of suspected OSAHS patients who were treated in the Department of respiration in the First Affiliated Hospital of Jinan University in January 2016, -2018, were randomly divided into two groups, with 120 cases in each group. The reasons for the treatment were daytime sleepiness, nocturnal snoring or suffocating or suffocating. 
After admission, the patients were all diagnosed with polysomnographic surveillance. The patients were informed and agreed by the family members, and all of them were in compliance with the ethics committee. There was no statistical difference in the basic data, age and sex between the two groups $(\mathrm{P}>0.05)$ (Table 1).

Table 1. Comparison of data among groups.

\begin{tabular}{|c|c|c|c|c|c|c|c|}
\hline \multirow[b]{2}{*}{ Group } & \multirow[b]{2}{*}{ male/female } & \multirow{2}{*}{$\begin{array}{l}\text { Average age } \\
\text { (years) }\end{array}$} & \multirow{2}{*}{$\begin{array}{l}\text { Mean course of } \\
\text { illness (month) }\end{array}$} & \multicolumn{3}{|l|}{ complications } & \multirow{2}{*}{$\begin{array}{l}\text { Body mass } \\
\text { index }\left(\mathrm{kg} / \mathrm{m}^{2}\right)\end{array}$} \\
\hline & & & & $\begin{array}{l}\text { High blood } \\
\text { pressure }(\%)\end{array}$ & $\begin{array}{l}\text { Coronary heart } \\
\text { disease (CHD) (\%) }\end{array}$ & Arrhythmia. (\%) & \\
\hline $\begin{array}{l}\text { Observation } \\
\operatorname{group}(n=120)\end{array}$ & $64 / 56$ & $54.3 \pm 5.4$ & $7.3 \pm 1.8$ & $10(8.3 \%)$ & $6(5.0 \%)$ & $4(3.3 \%)$ & $22.8 \pm 2.3$ \\
\hline $\begin{array}{l}\text { The control } \\
\text { group }(n=120)\end{array}$ & $62 / 58$ & $55.2 \pm 4.2$ & $7.1 \pm 1.6$ & $8(6.7 \%)$ & $5(4.2 \%)$ & $4(3.3 \%)$ & $23.7 \pm 2.8$ \\
\hline$\chi^{2} / \mathrm{t}$ & 0.256 & 0.312 & 0.305 & 0.402 & 0.256 & 0.322 & 0.245 \\
\hline
\end{tabular}

\subsection{Standards}

Inclusion criteria (1) is 20 to 75 years old, gender is not limited; (2) the monitoring time of patients with similar clinical symptoms of OSAHS was longer than $7 \mathrm{~h}$ (3) informed consent was obtained.

Exclusion criteria (1) severe or severe organ failure; (2) with acromegaly, hypothyroidism; (3) nocturnal staff or a clear history of neurasthenia; (4) patients with consciousness disorder or history of psychosis; (5) did not cooperate with nursing care.

\subsection{Nursing Method}

The control group carried out nursing care according to the routine nursing method of PSG monitoring, including informing the patients of the monitoring method and matters needing attention before operation, answering the patients' questions and observing the monitoring instruments during the monitoring. Timely adjustment should be made when there are problems, and the patients diagnosed with OSAHS should be informed of their prognosis and positive treatment methods, as well as the matters needing attention in life [6].

The observation group was given comprehensive nursing intervention during the peri-monitoring period. The specific methods were as follows:

(1) Education and propaganda. 1. The purpose, principle, process and matters needing attention of PSG monitoring were explained to the patients in detail. In the form of a brochure (including monitoring the day's diet, the amount of sleep during the day, the preparation of the bath before monitoring) and video playing, the patients were given specific knowledge of the monitoring process of PSG, so as to guide the patients to fully prepare for the monitoring. Start monitoring at $9 \mathrm{pm}$ to inform patients of daytime diet and exercise methods, avoid irritating diet, and eat less for dinner. Practice the method of urinating in bed. Inform the patients of the pathogenesis, prognosis, significance of early diagnosis and treatment of OSAHS [7]. B Psychological intervention: the anxiety degree of patients was evaluated by SAS anxiety scale on admission, and the patients were familiar with the monitoring facilities and environment, so as to improve the familiarity of the patients. While introducing the relevant procedure in detail for those with obvious anxiety, listen patiently, answer doubts and inform the patient to relax, which can properly direct the patient to deep breathing and muscle relaxation to relax the muscles. Inform the patient of the safety of monitoring process, can rest assured to sleep, like at home. C environmental facilities: ensure sleep monitoring room comfortable, clean, light and sound insulation effect, and the room facilities, such as bedside call system, first aid equipment, etc. Can be provided with an escort bed to accompany the patient. Understand patients sleep habits, such as pillows, mattress requirements, sleep posture, bedding color soft, tell patients can also bring their own pillows and sheets, as far as possible to meet the needs of patients, create a warm environment, so that patients have a feeling of home, Patients were given 3 days of psychological adaptation.

(2) In the process of monitoring. Correct placement of electrodes and leads to inform patients that there is no psychological burden, can be adjusted at will, abscission electrodes or lead, will be found and treated in time, fixed electrodes should use a good permeability and viscosity of $3 \mathrm{M}$ rubber cloth, improve the comfort of patients; The oxygen saturation monitor should be fixed on the comfort of the patient and be as firm as possible; ensure that the nasal airflow sensor catheter flows without twists and turns; the chest and abdomen straps should adjust the tightness reasonably on the basis of showing the images, and the connecting lines should be arranged neatly. Try to keep one or two bundles next to the pillow and set aside enough length to protect the patient from the change of position. For the peak time of nocturnal sleep apnea (such as 2: 5 in the second half of the night), the responsible nurse strengthens the inspection, moves gently during the inspection, avoids disturbing the patient [8].

(3) The monitoring is over. In the process of removing the monitoring instrument, gently wipe and paste the skin with warm towel, or massage slightly to promote the recovery of local blood circulation and increase the comfort. And pay attention to the psychological comfort of patients, inform patients that the monitoring has been completed. The patients diagnosed with OSAHS were informed of the importance of early treatment and the matters needing attention in life, such as proper exercise, weight reduction, smoking and alcohol withdrawal, sleeping in lateral position as far as possible, reducing the possibility of upper airway obstruction, and forming a good sleep habit [9]. 


\subsection{Observation Indicators}

Monitoring effect: the diagnostic rate of OSAHS was observed, the monitoring success rate of 9 indexes monitored by S1 S4: PSG was more than 4 hours at the same time, the sum of S1 S4 and REM sleep period was more than 4 hours, the success rate of $\mathrm{S} 1 \sim \mathrm{S} 4$ and REM was equal to the number of successful monitoring cases / the total number of people $\times$ 100. The diagnostic criteria of OSAHS were apnea hypopnea index (AHI) more than 5 times per hour.

Comfort score. According to the comfort status scale (GCQ), the scale consists of four dimensions, which are physical, psychological, spiritual, sociocultural and environmental, with 28 items. The scale used 1-4 Liken Scale scoring method to indicate strong disapproval. Negative item 1 indicates that you agree with me very much and that you disagree with me very much. The higher the score, the more comfortable it is. At the beginning of the monitoring, the monitoring is completed once each assessment.

Changes of anxiety. At admission and at the beginning of monitoring, the anxiety degree of the patients was evaluated after the monitoring, and 20 items were evaluated with SAS anxiety scale. The standard score was above 50 points for anxiety, and the standard score $=$ total score $\times 1.25$, and the integral part was used. Anxiety was positively correlated with scores.

The patient's satisfaction with the nursing care was used to investigate the satisfaction of the two groups of patients on the day of discharge from the hospital, including the service attitude, education and publicity, business ability, answers and guidance of the nursing workers. $>90$ points, satisfaction is $80-89$ points, generally $60-79$ points, unsatisfactory is $\leq 59$ points. Total satisfaction $=$ (very satisfactory number of cases + number of satisfied cases) / total number of cases $\times 100 \%$.

\subsection{Statistical Analysis}

Statistical analysis was carried out using SPSS 17.0, and the measurement data were normally distributed. The mean +1 -standard deviation (mean $+/$-s) indicated that the comparison between groups was conducted by $t$ test, while the comparison of the counting data was conducted by using the cat 2 test, $\mathrm{P}<0$. 05 is statistically significant.

\section{Results}

The monitoring success rate of the monitoring effect observation group was $95.0 \%(114 / 120)$ and OSAHS positive rate was $57.0 \%(65 / 114)$, which was lower than $71.7 \%$ $(82 / 116)$ of the control group and $\mathrm{P}<0.05$. Among the causes of monitoring failure, 4 patients in the control group $(3.3 \%)$ with short sleep duration were higher than 2 patients in the observation group $(\mathrm{P}<0.05)$ (table 2$)$.

Table 2. Comparison of monitoring effect between the two groups [n, (\%)].

\begin{tabular}{lllll}
\hline \multirow{2}{*}{ Group } & Monitoring success & $\begin{array}{l}\text { OSAHS positive } \\
\text { rate (\%) }\end{array}$ & \multicolumn{2}{l}{ Reason for failure } \\
\cline { 3 - 5 } & $116(96.7 \%)$ & $82(70.7 \%)$ & $2(1.7 \%)$ & $1(0.8 \%)$ \\
Short sleep (\%) & Electrode loss (\%) & $\begin{array}{l}\text { Blood oxygen saturation } \\
\text { drops off (\%) }\end{array}$ \\
\hline Observation group $(\mathrm{n}=120)$ & $114(95.0 \%)$ & $65(57.0 \%)$ & $4(3.3 \%)$ & $2(1.7 \%)$ \\
The control group $(\mathrm{n}=120)$ & 10.253 & 11.245 & 8.245 & 6.235 \\
$\chi 2 / \mathrm{t}$ & $<0.05$ & $<0.05$ & $<0.05$ & $<0.05$ \\
$\mathrm{P}$ & & & & 0 \\
\hline
\end{tabular}

The GCQ score ratio of the observation group was $(46.2+5.2)(53.6+4.1)$ at the beginning and the end of the comfort condition monitoring, which was higher than that of the control group $(\mathrm{P}<0.05)$ (table 3$)$.

Table 3. Comparison of comfort between the two groups of patients (score, mean $\pm S D)$.

\begin{tabular}{lll}
\hline group & At the beginning of monitoring & When monitoring is complete \\
\hline Observation group $(\mathrm{n}=120)$ & $46.2 \pm 5.2$ & $53.6 \pm 4.1$ \\
The control group $(\mathrm{n}=120)$ & $38.5 \pm 3.9$ & $44.5 \pm 5.7$ \\
$\mathrm{t}$ & 7.562 & 7.568 \\
$\mathrm{P}$ & $<0.05$ & $<0.05$ \\
\hline
\end{tabular}

There was no statistically significant difference in anxiety level between the two groups at admission $(\mathrm{P}>0.05)$. At the beginning of monitoring, the anxiety level of the observation group was significantly decreased, which was better than that of the control group $(\mathrm{P}<0.05)$. There was no significant change in anxiety level in the control group ( $\mathrm{P}>0$. 05); After the end of the monitoring, the anxiety level of both groups decreased, and the anxiety level of the observation group was better than that of the control group $(\mathrm{P}<0.05)$ (table 4$)$.

Table 4. Anxiety score of patients between groups (mean $+S D)$.

\begin{tabular}{llll}
\hline \multirow{2}{*}{ Group } & Anxiety score & & \\
\cline { 2 - 3 } & On admission & At the beginning of monitoring & When monitoring is complete \\
\hline Observation group $(\mathrm{n}=120)$ & $52.3 \pm 2.1$ & $46.5 \pm 2.3$ & $42.8 \pm 2.5$ \\
The control group $(\mathrm{n}=120)$ & $54.6 \pm 1.9$ & $50.3 \pm 2.2$ & $48.7 \pm 2.4$ \\
$\mathrm{t}$ & 0.425 & 7.562 & 4.256 \\
$\mathrm{P}$ & $>0.05$ & $<0.05$ & $<0.05$ \\
\hline
\end{tabular}


The nursing satisfaction of patients in the observation group (95.0\%) was significantly higher than that of the control group $(85.8 \%), \mathrm{P}<0.05$ (table 5).

Table 5. Comparison of nursing satisfaction between the two groups.

\begin{tabular}{|c|c|c|c|c|c|}
\hline Group & Very satisfied with & satisfaction & general & Not satisfied with & Satisfaction (\%) \\
\hline Observation group $(n=120)$ & 71 & 43 & 5 & 1 & 95.0 \\
\hline The control group $(n=120)$ & 58 & 45 & 11 & 6 & 85.8 \\
\hline$\chi^{2}$ & & & & & 11.235 \\
\hline
\end{tabular}

\section{Discussion}

OSAHS is a common disease in respiratory medicine [10]. When sleeping at night, intermittent apnea leads to hypoxia of patients, reduces sleep quality, and affects their normal work and life [11]. The persistence of OSAHS can lead to the disorder of patients' body metabolism, increase the risk of cardiovascular, cerebrovascular and other diseases, and pose a potential threat to patients' life safety [12]. PSG monitoring can diagnose OSAHS at an early stage and directly determine OSAHS degree. Accurate data are helpful for the setting of clinical treatment programs [13]. However, under the influence of various factors, the success rate of PSG monitoring is reduced. It has been reported that the failure rate of PSG monitoring can reach $10 \%$ to $15 \%$, thus affecting OSAHS diagnosis rate. Based on the combination of clinical practice and data review, the author analyzes the effective factors influencing PSG monitoring, which are poor sleep quality and shortened sleep time after patients change the environment [14]. PSG monitoring requires more than $7 \mathrm{~h}$ and takes longer time, which affects the success rate of monitoring. The reason for the shortened sleep time of the patients may be due to the change of sleep environment, strong strangeness, easy emotional anxiety after admission, and fear of their own degree of illness [15]. In addition, poor cognition of the monitoring process is also the cause of excessive anxiety and shortened sleep time. Therefore, it is of vital importance to provide patients with thoughtful and comprehensive peri-monitoring nursing intervention. Guided by the comprehensive nursing concept, "people-oriented" is given full play to provide patients with humanized services. The nursing work is not only comprehensive and meticulous, but also meets patients' personal needs as far as possible [16].

The nursing work in this study focused on the setting of monitoring environment, striving to provide patients with an environment like the sleeping conditions at home, and giving patients a psychological adjustment period of 3 days, reducing strangeness and avoiding the first-night effect of monitoring [17]. At the same time, education publicity and meticulous monitoring and nursing care can improve patients' comfort level, relieve patients' anxiety during monitoring, strive for natural sleep for patients, and improve the authenticity of results. Some scholars believe that providing patients with comfortable care can effectively improve their comfort level [18]. This study showed that the monitoring success rate of the observation group was significantly improved, and only 2 patients failed to monitor due to short sleep time, while 4 patients in the control group showed no difference in the factors of electrode shedding and oxygen saturation shedding, indicating that nursing intervention could effectively improve the sleep condition of patients [19]. At the same time, the degree of comfort and anxiety of patients in the observation group was significantly better than that of the control group, which also indicated the value of nursing intervention, improved the satisfaction degree of patients to nursing work, and promoted the establishment of a harmonious medical environment [20].

\section{Conclusion}

Comprehensive nursing intervention in the peri-monitoring period can effectively improve the success rate of PSG in OSAHS monitoring, improve the comfort level of patients in the monitoring process, and thus improve patients' satisfaction with nursing work.

\section{References}

[1] Chen QR, Dai QQ, Zou J, Zheng H. Crouzon syndrome coupled with OSAHS and congenital heart disease: a case report. Journal of clinical otorhinolaryngology, head, and neck surgery. 2018; 32(10):787-8.

[2] Zhou SL, Meng B, Ding JH. The expression of serum hepatocyte growth factor in OSAHS. Journal of clinical otorhinolaryngology, head, and neck surgery. 2017; 31(9):690-3.

[3] Peng Y, Zhou L, Cao Y, Chen P, Chen Y, Zong D, et al. Relation between serum leptin levels, lipid profiles and neurocognitive deficits in Chinese OSAHS patients. The International journal of neuroscience. 2017; 127(11):981-7.

[4] Yan YR, Lin YN, Li QY. Heterogeneity and individualized treatment of OSAHS: current status and prospects. Chinese journal of tuberculosis and respiratory diseases. 2018; 41(6):494-6.

[5] Zhao J, Han S, Zhang J, Wang G, Wang H, Xu Z, et al. Association between mild or moderate obstructive sleep apnea-hypopnea syndrome and cognitive dysfunction in children. Sleep medicine. 2018; 50:132-6.

[6] Scartabelli G, Querci G, Marconi L, Ceccarini G, Piaggi P, Fierabracci P, et al. Liver Enlargement Predicts Obstructive Sleep Apnea-Hypopnea Syndrome in Morbidly Obese Women. Frontiers in endocrinology. 2018; 9:293. 
[7] Paoloni V, Cretella Lombardo E, Placidi F, Ruvolo G, Cozza P, Lagana G. Obstructive sleep apnea in children with Marfan syndrome: Relationships between three-dimensional palatal morphology and apnea-hypopnea index. International journal of pediatric otorhinolaryngology. 2018; 112:6-9.

[8] Liu YT, Zhang HX, Li HJ, Chen T, Huang YQ, Zhang L, et al. Aberrant Interhemispheric Connectivity in Obstructive Sleep Apnea-Hypopnea Syndrome. Frontiers in neurology. 2018; 9:314.

[9] Matsui K, Sasai-Sakuma T, Ishigooka J, Inoue Y. Insufficient sleep rather than the apnea-hypopnea index can be associated with sleepiness-related driving problems of Japanese obstructive sleep apnea syndrome patients residing in metropolitan areas. Sleep medicine. 2017; 33:19-22.

[10] Garcia-Campos E, Labra A, Galicia-Polo L, Sanchez-Narvaez F, Haro R, Jimenez U, et al. Decrease of respiratory events in patients with obstructive sleep apnea-hypopnea syndrome using a mandibular advancement device assessed with split night polysomnography. Sleep Sci. 2016; 9(3):221-4.

[11] Agha B, Johal A. Facial phenotype in obstructive sleep apnea-hypopnea syndrome: a systematic review and meta-analysis. Journal of sleep research. 2017; 26(2):122-31.

[12] Chen T, Yang M, Liu B, Liu YT, Zhang HX, Liu CC, et al. The Resting-State Functional Connectivity of the Default Mode Networks in Patients With Obstructive Sleep Apnea-Hypopnea Syndrome. CNS \& neurological disorders drug targets. 2016.

[13] Hang LW, Chen CF, Wang CB, Wu TN, Liang WM, Chou TC. The association between continuous positive airway pressure therapy and liver disease development in obstructive sleep apnea/hypopnea syndrome patients: a nationwide population-based cohort study in Taiwan. Sleep \& breathing = Schlaf \& Atmung. 2017; 21(2):461-7.

[14] Liu Y, Gu YC, Tang Y, Lu XL, Chen H, Wu QZ. Role and mechanism of Th17/Treg in obstructive sleep apnea hypopnea syndrome. Zhonghua yi xue za zhi. 2016; 96(44):3577-81.
[15] Alvarez D, Kheirandish-Gozal L, Gutierrez-Tobal GC, Crespo A, Philby MF, Mohammadi M, et al. Automated analysis of nocturnal oximetry as screening tool for childhood obstructive sleep apnea-hypopnea syndrome. Conference proceedings: Annual International Conference of the IEEE Engineering in Medicine and Biology Society IEEE Engineering in Medicine and Biology Society Annual Conference. 2015; 2015:2800-3.

[16] Huai D, Dai J, Xu M, Cao Y, Song H, Wang S, et al. Combination of $\mathrm{CO} 2$ laser-assisted uvulopalatopharyngoplasty and nasal cavity expansion enhances treatment of obstructive sleep apnea-hypopnea syndrome. International journal of clinical and experimental medicine. 2015; 8(10):19764-74.

[17] Mosharraf-Hossain AK, Ahmed K, Islam MT, Chakrabortty R. A Community study of obstructive sleep apnea hypopnea syndrome (OSAHS) in middle-aged Bangladeshi population. Bangladesh Medical Research Council bulletin. 2015; 41(1):13-8.

[18] Wu W, Li Z, Tang T, Wu J, Liu F, Gu L. 5-HTR2A and IL-6 polymorphisms and obstructive sleep apnea-hypopnea syndrome. Biomedical reports. 2016; 4(2):203-8.

[19] Wang Q, Chen R, Cao Z, Yuan H, Zhao W, Li X, et al. Effects of simultaneous multi-level surgery intervention on the changes of the oxidative stress and the cognitive function in young and middle-aged patients with Moderate-severe obstructive sleep apnea hypopnea syndrome. Journal of clinical otorhinolaryngology, head, and neck surgery. 2015; 29(24):2139-42.

[20] Chen X, Zhou T, Li D, Zhang C, Jia P, Ma J, et al. Evaluating the clinical value of oscillatory cardiopulmonary coupling in patients with obstructive sleep apnea hypopnea syndrome by impedance cardiogram. Sleep medicine. 2016; 19:75-84. 